\title{
Experimental investigation of relative timing jitter in passively synchronized Q- switched lasers
}

Cheng, Haynes Pak Hay; Tidemand-Lichtenberg, Peter; Jensen, Ole Bjarlin; Andersen, Peter Eskil; Petersen, Paul Michael; Pedersen, Christian

Published in:
Optics Letters

Link to article, DOI:

10.1364/OL.36.000415

Publication date:

2011

Document Version

Publisher's PDF, also known as Version of record

Link back to DTU Orbit

Citation (APA):

Cheng, H. P. H., Tidemand-Lichtenberg, P., Jensen, O. B., Andersen, P. E., Petersen, P. M., \& Pedersen, C. (2011). Experimental investigation of relative timing jitter in passively synchronized Q-switched lasers. Optics Letters, 36(3), 415-417. https://doi.org/10.1364/OL.36.000415

\section{General rights}

Copyright and moral rights for the publications made accessible in the public portal are retained by the authors and/or other copyright owners and it is a condition of accessing publications that users recognise and abide by the legal requirements associated with these rights.

- Users may download and print one copy of any publication from the public portal for the purpose of private study or research.

- You may not further distribute the material or use it for any profit-making activity or commercial gain

- You may freely distribute the URL identifying the publication in the public portal 


\title{
Experimental investigation of relative timing jitter in passively synchronized $Q$-switched lasers
}

\author{
Haynes Pak Hay Cheng, * Peter Tidemand-Lichtenberg, Ole Bjarlin Jensen, \\ Peter Eskil Andersen, Paul Michael Petersen, and Christian Pedersen \\ DTU Fotonik, Technical University of Denmark, DK-400o Roskilde, Denmark \\ ${ }^{*}$ Corresponding author: hach@fotonik.dtu.dk
}

Received November 3, 2010; revised December 22, 2010; accepted December 23, 2010; posted January 7, 2011 (Doc. ID 137631); published January 31, 2011

\begin{abstract}
Relative timing jitter between synchronized $Q$-switched lasers, or lack thereof, is important for stable sum-frequency generation. Experimental investigation of two passively synchronized lasers shows that the jitter is minimized when the free-running repetition rates of the two lasers are close to, but not exactly, matching. When the free-running repetition rates are matched, the jitter is significantly large. At the best operating point, the pulse-to-pulse period was $200 \mu \mathrm{s}$, while the relative jitter between the two lasers was $9 \mathrm{~ns}$. If the effect of the master laser's pulse-to-pulse jitter is removed, the residual timing jitter between the two lasers was $6 \mathrm{~ns}$, which corresponds to the lower limit set by pump power fluctuations and noise from spontaneous emission. @ 2011 Optical Society of America

OCIS codes: $140.3540,140.3580$.
\end{abstract}

Timing jitter in passively synchronized $Q$-switched lasers is of concern in many applications, such as sumfrequency generation and pump-probe experiments. The dominant cause of timing jitter in a single passively $Q$-switched laser has been attributed to pump power fluctuations [1] and noise from amplified spontaneous emission [2]. Subsequently, jitter reduction methods that increases gain modulation [1] or loss modulation [2-4 ] or self-injects a seed pulse $[\bar{b}]$ at close to the laser's repetition rate have been proposed. In particular, direct bleaching of the saturable absorber (SA) by a laser diode bar resulted in a 12 times decrease in timing jitter [4], while a self-injection seeded microchip laser achieved a 600 times reduction [5]. Loss modulation was applied at a slightly higher repetition rate than the laser's natural free-running repetition rate, just before the laser reaches threshold [3,4].

Synchronized $Q$ switching have previously been demonstrated in both active [6,7] and passive [을 12$]$ systems. Notably, two lasers are mode-matched such that the higher-repetition rate (master) laser bleaches the SA before the lower-repetition rate (slave) laser reaches threshold [9-11]. However, to the authors' knowledge, no work has been done on the detailed investigation of timing jitter in synchronized $Q$ switching, in particular, the relative timing jitter between the two synchronized pulse trains. This is particularly important in the application of sum-frequency generation (SFG), where accurate timing of the two pulses is critical for stability in their temporal overlap and in the resulting SFG power.

In this Letter, an experimental investigation into the relative timing jitter between two passively synchronized lasers is presented, while a qualitative numerical model confirms the experimental results-there exists a tradeoff between minimal relative delay and minimal relative jitter. Furthermore, the penalty, in terms of timing jitter, for using a passively synchronized system instead of an active system is determined from experimental results. The relative jitter between the two passively synchronized lasers was $9 \mathrm{~ns}$ at the best operating point. By sub- tracting the effect of pulse-to-pulse jitter in the master laser, it is estimated that the relative jitter of a similar actively synchronized system could be no better than $6 \mathrm{~ns}$, highlighting the lower limit set by pump power fluctuations and noise from amplified spontaneous emission.

The experimental setup is illustrated in Fig. 1, while detailed coating specifications and cavity parameters can be found in [11]. The high reflection facet of Nd:YAG1, mirrors M1, BS, M2, and the intracavity lens LS form the $946 \mathrm{~nm}$ laser cavity, while the high reflection facet of Nd:YAG2 and mirrors M3 and M2 form the $1064 \mathrm{~nm}$ laser cavity. BS is the coupling mirror coated for high transmission at $1064 \mathrm{~nm}$ and high reflection at $946 \mathrm{~nm}$. Synchronized $Q$ switching is achieved by placing one SA, a Cr:YAG crystal specified for $15 \%$ small-signal absorption at normal incidence at $1064 \mathrm{~nm}$, in the common section between mirrors BS and M2. The 946 nm laser cavity was made relatively long to ensure a good temporal overlap between the two pulse trains. The cavity lengths were $37 \mathrm{~cm}$ and $19 \mathrm{~cm}$ for the $946 \mathrm{~nm}$ and $1064 \mathrm{~nm}$ lasers, respectively, and the resulting pulse widths (FWHM) were $200 \mathrm{~ns}$ and $45 \mathrm{~ns}$, respectively. Incident pump power of the $946 \mathrm{~nm}$

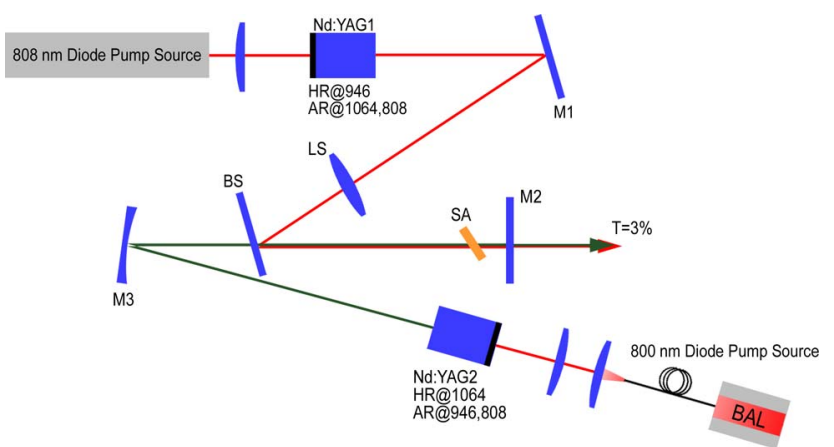

Fig. 1. (Color online) Experimental setup for synchronized $Q$ switching. Nd:YAG1 and Nd:YAG2 are the laser crystals for $946 \mathrm{~nm}$ and $1064 \mathrm{~nm}$, respectively. SA is the saturable absorber that is shared between the two lasers. The 946 and $1064 \mathrm{~nm}$ laser cavity lengths are 37 and $19 \mathrm{~cm}$, respectively. 
laser was held fixed at $1.6 \mathrm{~W}$, while incident pump power of the $1064 \mathrm{~nm}$ laser was varied.

Following the definition in $[\underline{4}, \underline{5}]$, timing jitter in this Letter is 1 standard deviation of the experimental data. Statistical measurements of the relative delay between the $1064 \mathrm{~nm}$ pulses and $946 \mathrm{~nm}$ pulses are obtained over 1000 samples. It will be confirmed below that the timing jitter follows a Gaussian distribution.

Figure 2(a) and 2(b) show, respectively, the relative delay and relative timing jitter between the two lasers as a function of the $1064 \mathrm{~nm}$ laser incident pump power. The $1064 \mathrm{~nm}$ laser is lagging the $946 \mathrm{~nm}$ laser when its pump power is below the switching point at $1.6 \mathrm{~W}$ and becomes the leading laser when its pump power is above the switching point. The delay between the two pulse trains is the smallest at the switching point, when the free-running repetition rates of the two lasers are equal. However, as analogous to the jitter reduction techniques reported in [3,4] for a single laser, the jitter becomes significantly large if loss modulation (by the master laser in this case) is equal to the slave laser's free-running repetition rate- or to be precise, when the free-running repetition rates of both lasers are equal. As both lasers build up at the same rate, both are above threshold when the SA is bleached; thus, small changes in the photon flux, amplified by stimulated emission, have a large impact on the buildup time and, consequently, on the relative timing of the two pulses [11]. This instability could be avoided in actively synchronized systems if the active $Q$ switch is cycled at a higher repetition rate than the free-running repetition rates of both lasers. The unstable operating regime in Fig. 2(b) corresponds to a range of tens of milliwatts of incident pump power. The minimum jitter on either side of the switching point in Fig. 2(b) then corresponds to an optimal operating point, where both the delay and the jitter would be the lowest for stable operation. Figure 2(c) is one frame from the uploaded video (Media 1), showing the change in the delay statistical distribution as the $1064 \mathrm{~nm}$ laser pump power is increased.

Adapting the coupled rate equations described in [10] to a three-level system, the theoretical delay and jitter are shown qualitatively in Figs. 3(a) and 3(b). The model assumes fluctuations of $0.5 \%$ in the $1064 \mathrm{~nm}$ laser absorbed pump power and $0.05 \%$ in the $946 \mathrm{~nm}$ laser absorbed pump power. The smaller fluctuation of the $946 \mathrm{~nm}$ laser absorbed pump power can be justified by the narrow spectrum pump source used. While the rate equations in [10] show a one-dimensional model that considers only the temporal dynamics of a spatially uniform field, the
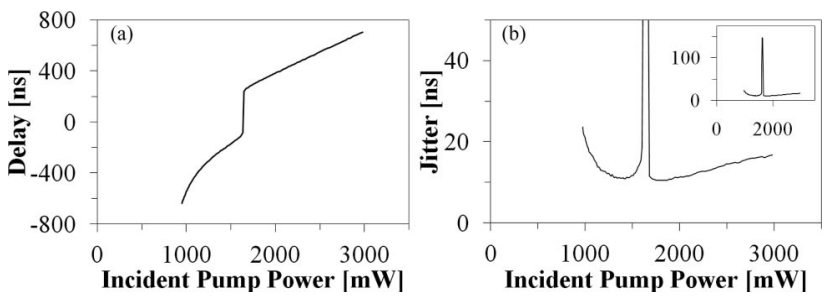

Fig. 3. Modeling results on (a) the delay and (b) relative timing jitter of the 946 and $1064 \mathrm{~nm}$ pulses. The timing jitter in (b) is plotted in the same scale as Fig. 2(b), and the inset shows the full scale.

steep slope in the resulting delay curve and the minimum jitter observed at around the switching point matches well with the experimental data. The inset of Fig. 3(b) shows the full scale of the timing jitter numerical results, where it can be seen that the large timing jitter at the switching point increases to $150 \mathrm{~ns}$. This was not observed in the experiments, possibly due to the high sensitivity in the unstable region, and the difficulty in maintaining the laser pump power at exactly the switching point.

To investigate the increase in timing jitter when working with passively synchronized lasers, as opposed to actively synchronized systems, one can examine the relative timing jitter between the two lasers as a function of the pulse-to-pulse timing jitter in the master laser. This is shown in Fig. 4(a), where the inset shows graphically the measurement that is taken. The incident pump power of the $1064 \mathrm{~nm}$ laser was $1.58 \mathrm{~W}$; thus the $946 \mathrm{~nm}$ laser was the master laser at this operating point. Projecting the data points in Fig. 4(a) onto the vertical axis, a histogram of the relative delay between the synchronized pulses can be obtained and is shown in Fig. 4(b). The mean relative delay and relative jitter between the two lasers were 119 ns and 9 ns, respectively. Similarly, the master laser pulse-to-pulse separation and jitter were $202 \mu \mathrm{s}$ and $4 \mu \mathrm{s}$, respectively, and the corresponding histogram is shown in Fig. 4(c). The solid lines in Figs. 4(b) and 4(c) show good fits to the Gaussian distribution.

The solid line in Fig. 4(a) is the least-square fit, which shows the correlation between the pulse-to-pulse separation and the relative delay between the two synchronized pulses. This was found to be $1.3 \mathrm{~ns} / \mu \mathrm{s}$. Subtracting this fitted line from the experimental data removes the effect of pulse-to-pulse jitter in the master laser and maps the delay onto the horizontal axis. This is shown in Fig. 4(d). By projecting the data points in Fig. 4(d) onto the vertical axis, the residual relative jitter is plotted as a histogram in
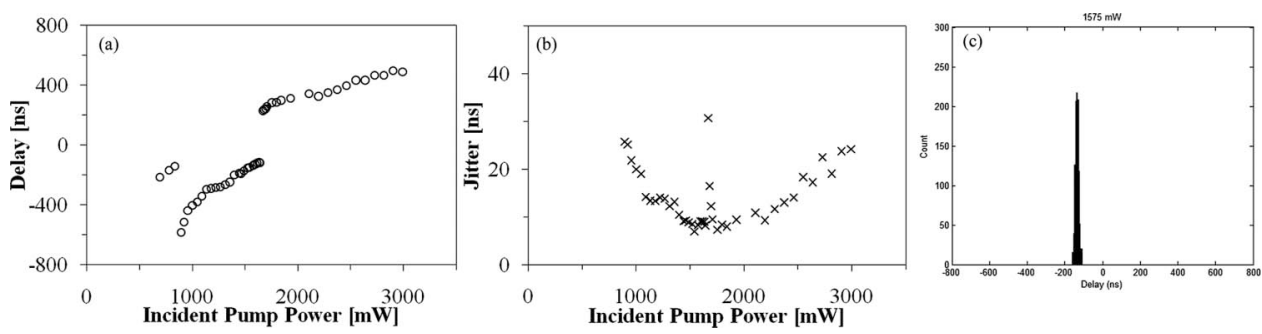

Fig. 2. (a) Delay and (b) timing jitter of the 946 and $1064 \mathrm{~nm}$ pulses relative to each other as a function of the $1064 \mathrm{~nm}$ laser pump power, with the $946 \mathrm{~nm}$ laser incident pump power held fixed at $1.6 \mathrm{~W}$. A negative delay indicates that the $1064 \mathrm{~nm}$ pulses were lagging the $946 \mathrm{~nm}$ pulses. (c) Single frame from the uploaded video (Media 1) showing the statistical distribution of the delay and the corresponding incident pump power of the $1064 \mathrm{~nm}$ laser. 

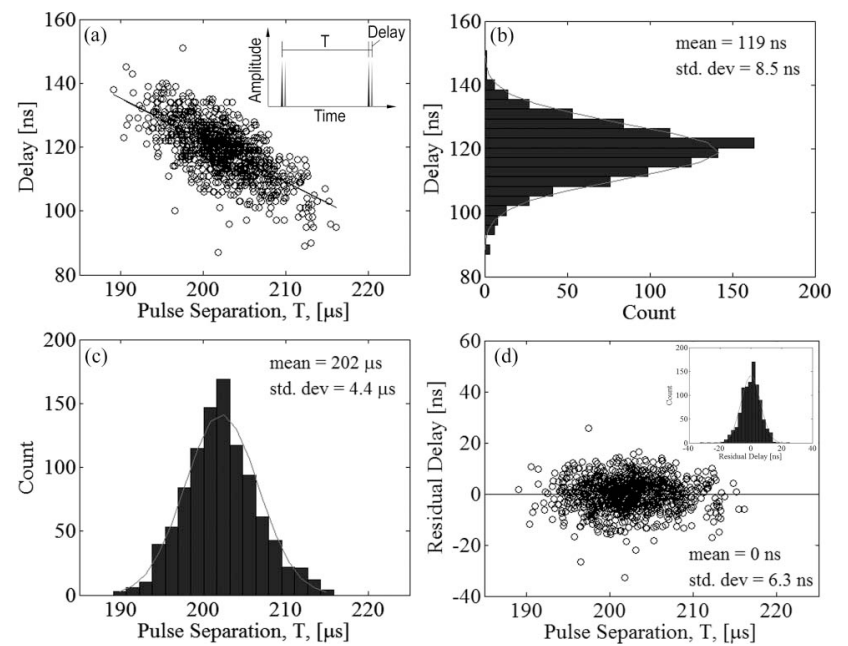

Fig. 4. (a) Correlation of pulse separation of the master laser and delay between the two lasers at a fixed pump power. The inset depicts the measurement that was carried out, while the solid line shows the least-square fit. Projection of the data points onto the vertical axis is shown as a histogram in (b), while projection of the data points onto the horizontal axis is shown as a histogram in (c). The slope of the least-square fit is subtracted from the experimental data. The resulting residual jitter, with the effect of the master laser's pulse-to-pulse jitter removed, is shown in (d), and the corresponding histogram is shown in the inset. Solid lines in (b) and (c) show the Gaussian fits.

the inset. The residual relative jitter between the two lasers, without the effect of pulse-to-pulse jitter in the master laser, is 6 ns.

Figure 4(d) implies that, if the pulse-to-pulse jitter in the master laser can be well controlled, as in the case of actively $Q$-switched systems, the expected relative jitter between the two lasers can be no better than 6 ns. This is the lower limit set by pump power fluctuations and noise from amplified spontaneous emission. Comparing this value to the 9 ns relative timing jitter currently observed in the experimental data, shows the penalty that is paid by using an all-passive system.

In conclusion, experimental investigation into the relative timing jitter between passively synchronized $Q$-switched lasers has been presented for the first time (to our knowledge). Results from a numerical model supported the experimental results and showed the detailed features that were observed experimentally. In particular, relative jitter between the two lasers would be the lowest at just before and after the unstable regime, where the free-running repetition rates of the two lasers are matched. These correspond to the optimal operating point where both the relative delay and relative jitter are the lowest for stable operation. Relative timing jitter between the passively synchronized lasers was $9 \mathrm{~ns}$ at the best operating point and increased significantly at the switching point. If the effect of pulse-to-pulse jitter in the master laser is removed, the residual jitter is $6 \mathrm{~ns}$, which is the lower limit that can be reached if a master laser with no pulse-to-pulse jitter was used.

This work was supported by the European Union FP-6 project WWW.BRIGHTER.EU, contract IST-2005-035266. The authors acknowledge Ferdinand-Braun-Institut, Leibniz Institut für Höchstfrequenztechnik for providing the tapered diode amplifier.

\section{References}

1. J. B. Khurgin, F. Jin, G. Solyar, C. C. Wang, and S. Trivedi, Appl. Opt. 41, 1095 (2002).

2. S. L. Huang, T. Y. Tsui, C. H. Wang, and F. J. Kao, Jpn. J. Appl. Phys. 38, L239 (1999).

3. X. Wang and Z. Xu, Appl. Opt. 45, 8477 (2006).

4. B. Cole, L. Goldberg, C. W. Trussell, A. Hays, B. W. Schilling, and C. McIntosh, Opt. Express 17, 1766 (2009).

5. A. Steinmetz, D. Nodop, A. Martin, J. Limpert, and A. Tünnermann, Opt. Lett. 35, 2885 (2010).

6. R. W. Farley and P. D. Dao, Appl. Opt. 34, 4269 (1995).

7. E. Herault, M. Lelek, F. Balembois, and P. Georges, Opt. Express 16, 19419 (2008).

8. L. Zhang, Z. Wei, B. Feng, D. Li, and Z. Zhang, Opt. Commun. 264, 51 (2006).

9. P. Tidemand-Lichtenberg, J. Janousek, R. Melich, J. L. Mortensen, and P. Buchhave, Opt. Commun. 241, 487 (2004).

10. J. Janousek, P. Tidemand-Lichtenberg, J. L. Mortensen, and P. Buchhave, Opt. Commun. 265, 277 (2006).

11. H. P.H. Cheng, P. Tidemand-Lichtenberg, O. B. Jensen, P. E. Andersen, P. M. Petersen, and C. Pedersen, Opt. Express 18, 23987 (2010).

12. T. Y. Tsai, Y. C. Fang, H. M. Huang, H. X. Tsao, and S. T. Lin, Opt. Express 18, 23523 (2010). 